\title{
Timeliness and QoS Aware Packet Scheduling for Next Generation Networks
}

\author{
Yao-Nan Lien and Yung-Chuan Wun \\ Department of Computer Science, National Chengchi University \\ Taipei, Taiwan, R.O.C. \\ lien@cs.nccu.edu.tw
}

\begin{abstract}
Running time sensitive multimedia services such as Voice-over-IP (VoIP) and Video-on-Demand (VoD) on All-IP Networks may have lower quality, i.e. Quality of Service (QoS), than its counterpart on conventional circuit-switched networks. We could enhance overall QoS without investing more resources if routers could forward packets based on their timeliness and QoS class giving important late packets proper precedence. This paper proposes a chargebased optimization model for packet scheduling aiming to maximize overall QoS satisfaction factor. We also developed a simple and effective scheduling policy based on this concept for the environments where each packet has a predefined hop-by-hop traveling schedule. Routers are assumed in three different queue architectures: ideal single preemptive queue and practical multiple FIFO queue with and without a priority queue. To forward a packet, a router first assigns an adequate profit function to the packet based on its timeliness and QoS class as well as the loading status of the succeeding routers along its traveling path, and then inserts the packet into an appropriate position in the output queues. The performance of this approach is evaluated by simulation using NS-2 network simulator. Simulation results show that, under heavy load, our approach can outperform easily the Simulated Priority Queue that differentiates QoS class only.
\end{abstract}

Keywords: Router, QoS, Scheduling, All-IP Network.

\section{INTRODUCTION}

An All-IP Network uses IP based packet-switched networks to carry all types of network traffics $[4,5]$. All-IP Network not only reduces network deployment and management costs, but also offers a great opportunity opening to various new services that are not possible on the conventional separated networks. Running time sensitive multimedia services such as Voice-over-IP (VoIP) and Video-on-Demand (VoD) on an All-IP Network may have lower quality than its counterpart on conventional circuit-switched networks. Influenced by many factors, the packets traveling on a packet-switched network may suffer from long delay time, large jitter and high packet loss rate. These problems may in turn impair the quality of timesensitive services. To improve QoS satisfaction factor, this paper proposes a new packet scheduling mechanism for routers to forward packets based on their timeliness and QoS class.

When a packet arrives its destination late, there is no way to correct the problem at the receiver side. Thus, it will be beneficial if routers could forward packets based on their timeliness and QoS class giving important late packets proper precedence, instead of using FIFO service plan or differentiating QoS class only. The overall QoS satisfaction factor will be improved without investing more resources.

This paper proposes a charge-based optimization model for packet scheduling aiming to maximize overall QoS satisfaction factor. We also developed a simple and effective scheduling policy based on this concept for the environments where each packet has a predefined hop-by-hop traveling schedule. To forward a packet, a router first assigns an appropriate profit function to the packet based on its timeliness and QoS class as well as the loading status of the succeeding routers along its traveling path, and then inserts the packet into an appropriate position in the output queues. The challenge is to find the best way to assign proper profit functions to different classes of packets in order to utilize resources more wisely, e.g. urgent and important packets get precedence. 


\subsection{Router Architecture}

A simplified router architecture is shown in Fig. 1. Incoming packets are put into an input queue. Packet by packet in FIFO fashion, an agent takes a packet out of the input queue, selects an output queue according to the decision given by the routing table, and put the packet into the selected output queue. Packets in each output queue will be transmitted to the next router. Our scheduling mechanism is applied to each of the output queues.

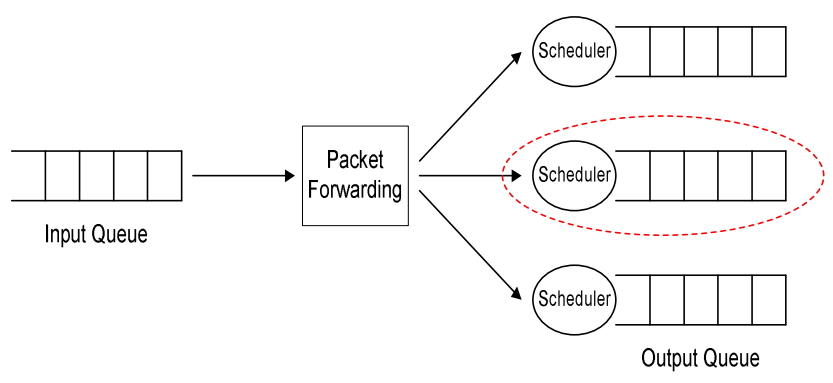

Fig. 1 Simplified Router Architecture

\subsection{Scheduling Architecture}

A simplified scheduling infrastructure for each output queue is shown in Fig. 2. Packet by packet, a scheduler put each incoming packet into an appropriate position in the output queue under the instruction of scheduling policy. In practice, an output queue may be decomposed into some FIFO queues for easy implementation.

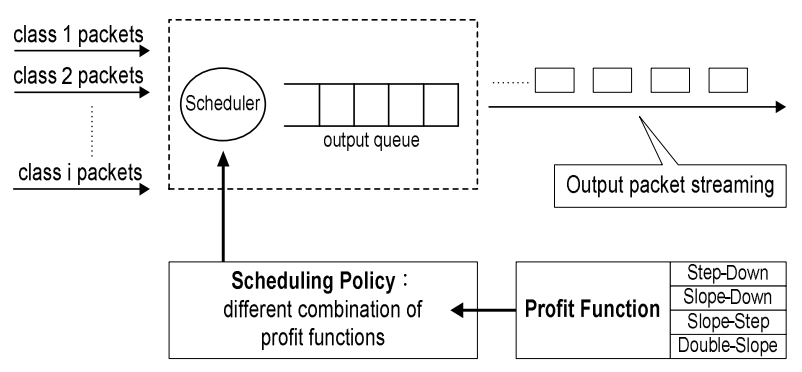

Fig. 2 Scheduling Mechanism in Router

\subsection{Packet Traveling Schedule}

In an ordinary packet-switched network, packet traveling is a stochastic process such that it is not easy to predict either the path it travels or its time schedule. However, to support All-IP Network QoS, some researches proposed to use designated path with allocated resources to deliver a packet [4]. Under this circumstance, there exists a time schedule for each packet. In this research, we assume each packet has a designated traveling path as well as a time schedule for our mechanism to be applicable.

\section{RELATED WORK}

A simple packet scheduling algorithm is FIFO scheduling, which treats all packets equally. If the network carries various traffic types with different characteristics and service requirements, a FIFO scheduling algorithm may perform poorly. To differentiate traffic types with different grade of service, more sophisticated scheduling algorithms are needed.

\subsection{Priority Queue}

A prioritized scheduling algorithm schedules packets delivery according to their priority, which can be calculated based on various criteria, such as QoS class. Priority Queue is a simple hardware implementation, which splits an output queue into several FIFO queues; an incoming packet is inserted into the tail end of a FIFO queue based on its priority; the scheduler then selects packets from these queues to serve. There are several ways to select packets to serve. The first one is "bandwidth sharing" style in which the scheduler selects the head of each queue in Round-Robin fashion, but higher priority queues get more turns than lower ones. The fraction of link bandwidth each queue obtained is proportional to its share in the round robin cycles. Typical scheduling disciplines of this style are Weighted Round-Robin and Weighted Fair Queueing. The second one is "preemptive" style in which higher priority queues are served before lower ones $[1,2,3,6,7,8]$.

In conventional Priority Queue approach, priority is determined by QoS class only and late packets do not get precedence. If timeliness and QoS are both taken into account in a prioritized scheduling, a better performance can be achieved.

\subsection{Path-Specific All-IP Networks and BBQ}

$B B Q$ is a QoS management infrastructure to support end-to-end QoS for All-IP Networks [4]. It takes budget-based approach to control the quality of each network component based on a calculated budget plan. End-to-end QoS will be assured by a global QoS 
management agent. The objective of this infrastructure is to facilitate network operators to tune their networks with a great flexibility and scalability to achieve their own operational objectives.

BBQ takes resource reservation approach to ensure QoS. A dedicate transmission path is assigned to each time-sensitive connection-oriented service request. Furthermore, the latency at each link is bounded by a designated budget. Therefore, there exists a hop-byhop traveling schedule for each packet. Our proposed timeliness and QoS aware packet scheduling is applicable to such an environment.

\section{TIMELINESS and QoS AWARE PACKET SCHEDULING}

\subsection{Scheduling Queue Architectures}

We assume three different output queue architectures. The first one is Simple Preemptive Queue (SPQ) as shown in Fig. 3. There is only one preemptive queue. A newly arrived packet can be inserted into any position of the queue. The second one is Multiple FIFO Queue (MQ) as shown in Fig. 4. An output queue is split into several FIFO queues. A newly arrived packet will be inserted into the tail end of a FIFO queue. A post scheduler selects a FIFO queue in round robin fashion to serve. When serving a FIFO queue, all packets are transmitted in FIFO fashion without preemption. The third one is Priority Multiple Queue (PMQ) as shown in Fig. 5. PMQ is similar to MQ, but with an additional priority queue, which has highest priority among all FIFO queues. Once there is any packet in the priority queue, it will be transmitted first. The packet with highest priority will be inserted into this queue.

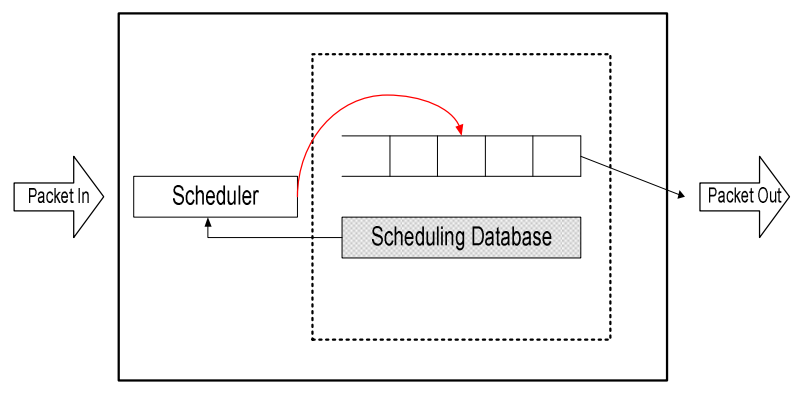

Fig. 3 Simple Preemptive Queue router

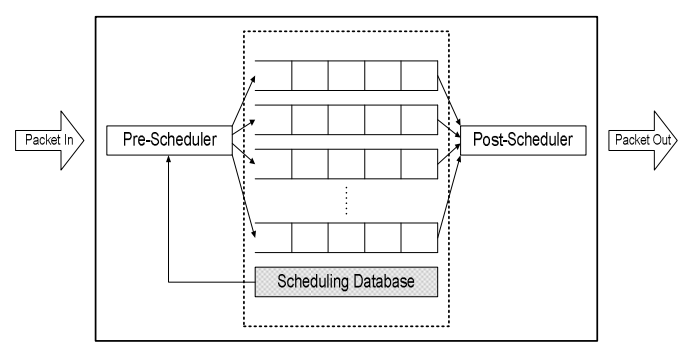

Fig. 4 Multiple FIFO Queue router

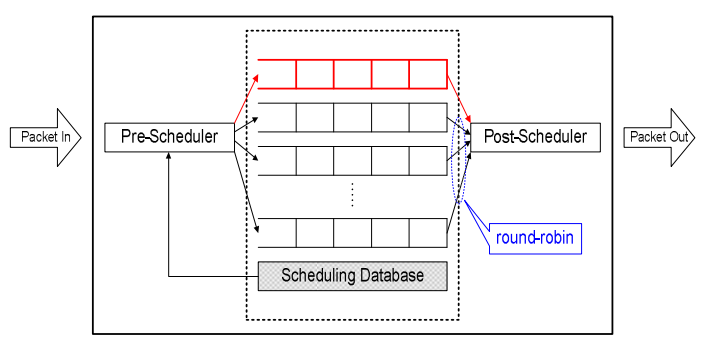

Fig. 5 Priority Multiple Queue router

The comparison of three different queue architectures is illustrated in Fig. 6. MQ can be viewed as a SPQ, but with only a limited number of insertable queuing positions, each corresponding to the tail of a FIFO queue, to insert new packets. Unfortunately, there is no insertable queueing position corresponding to the head of SPQ. In other words, even the most urgent packets must wait at least the length of a FIFO queue. On the other hand, PMQ has a priority queue that can be viewed as the head of SPQ so that the most urgent packets don't have to wait. In summary, SPQ has the best performance because a packet can be inserted into any position. MQ has the worst performance because it has the fewest insertable queuing positions. PMQ is better than MQ because it supports "no-wait" queuing position. Since the latency requirement for a router is very stringent, usually within $1 \mathrm{~ms}$, implementing SPQ may not be practical due to its hardware complexity. On the other hand, without preemption mechanism, MQ and PMQ are much easier to implement.

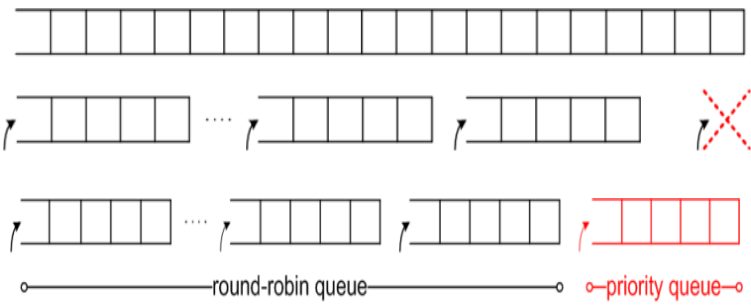

Fig. 6 Comparison of SPQ, MQ, and PMQ. 


\subsection{Optimization Model}

We assume there are three QoS classes: (1) Conversational, (2) Streaming, and (3) non-real-time. We propose an optimization model that takes QoS class, timeliness, and quality metrics into account. The objective of optimization model is shown in Eq. 1 and the notations are shown in Table 1. The tariff is sensitive to both packets and QoS class. A linear discount is applied to the tariff if the quality is impaired.

$$
\sum_{i=1}^{3} \sum_{j=1}^{N_{i}}\left(q_{i, j} \cdot C_{i} \cdot \lambda_{i, j}\right)
$$

Table 1 Notations of Objective Function

\begin{tabular}{|c|c|}
\hline Notation & Definition \\
\hline$N_{i}$ & number of flows of class $i$ \\
\hline$C_{i}$ & unit price per byte of class $i$ (tariff) \\
\hline \multirow{2}{*}{$\lambda_{i, j}$} & $\begin{array}{l}\text { number of bytes admitted in the flow } j \text { of class } i \\
\text { (Conversational or Streaming class) }\end{array}$ \\
\hline & $\begin{array}{l}\text { number of bytes received in the flow } j \text { of class } i \\
\text { (non-time-sensitive class) }\end{array}$ \\
\hline$q_{i, j}$ & $\begin{array}{l}\text { quality index of the flow } j \text { of class } i(0<=q<=1) \\
q_{i, j}=a_{i} \alpha_{i, j}+b_{i} \beta_{i, j}+c_{i} \gamma_{i, j}, \\
\text { if avg. delay time of flow } j \text { of class } i<=B_{i}{ }^{(1)} \\
\text { and avg. jitter of flow } j \text { of class } i<=B_{i}{ }^{(2)} \\
\text { and avg. loss rate of flow } j \text { of class } i<=B_{i}{ }^{(3)} \text {, } \\
q_{i, j}=0 \text {, otherwise. }\end{array}$ \\
\hline
\end{tabular}

The quality index of a flow of class $i$ is defined in Table 2. It is a linear combination of quality satisfaction factors of three quality metrics: delay time, jitter, and lost rate. Each of them has a minimum threshold. A flow is free of charge if any quality metric gets below the corresponding threshold.

Table 2 Notations of Quality Index

\begin{tabular}{|c|c|}
\hline Notation & Definition \\
\hline $\begin{array}{c}B_{i}^{(1)} \\
\left(B_{i}^{(2)}, B_{i}^{(3)}\right)\end{array}$ & $\begin{array}{l}\text { maximum acceptable average delay time } \\
\text { (jitter, loss rate) of class } i\end{array}$ \\
\hline$a_{i}\left(b_{i}, c_{i}\right)$ & $\begin{array}{l}\text { quality coefficient of delay time (jitter, loss } \\
\text { rate) of class } i\end{array}$ \\
\hline$\alpha_{i, j}$ & $\begin{array}{l}\text { delay time satisfaction factor of flow } j \text { of } \\
\text { class } i \text {; } \\
1 \text { - (avg. delay time of flow } j \text { of class } i) / B \\
(1)\end{array}$ \\
\hline$\beta_{i, j}$ & $\begin{array}{l}\text { jitter satisfaction factor of flow } j \text { of class } i \text {; } \\
1 \text { - (average jitter of flow } j \text { of class } i) / B_{i}^{(2)}\end{array}$ \\
\hline$\gamma_{i, j}$ & $\begin{array}{l}\text { loss rate satisfaction factor of flow } j \text { of } \\
\text { class } i \text {; } \\
1 \text { - (packet loss rate of flow } j \text { of class } i)\end{array}$ \\
\hline
\end{tabular}

\subsection{Scheduling Algorithms}

Packet scheduling can be either independent or dependent on the loading status of succeeding routers in the traveling path of a packet. The formal is called Intra-Router Scheduling (IRS) and the latter is called Look-Ahead Scheduling (LAS).

IRS scheduling takes two steps. For an incoming packet, the scheduling agent designates a profit function to the packet based on its timeliness and QoS class, and then calculates the best queuing position by enumerating all insertable queuing positions aiming to maximize the profit defined by the profit function, which will (hopefully) maximize the overall objective shown in Eq. 1.

LAS scheduling is similar to IRS scheduling, but LAS will modify the profit function according to the loading status of succeeding routers in the traveling path.

\subsection{Profit Functions}

The profit earned after transmitting a packet in a router is a function of transmission latency, which is approximately the waiting time in the output queue. Four different types are defined: Step-Down, SlopeDown, Slope-Step, and Double-Slope. Each type is represented by four parameters: soft-deadline $\left(T_{a}\right)$, hard-deadline $\left(T_{b}\right)$, pre-soft-deadline profit rate $\left(S_{1}\right)$, and post-soft-deadline profit rate $\left(S_{2}\right)$. Assuming $T_{d}$ is the time to transmit the packet (leaving the output queue), and the definitions of profit functions are shown in Table 3.

\subsection{Class-Aware Scheduling Policy}

In a multi-class system, resources must be allocated wisely to enhance overall QoS satisfaction factor if resources are limited. The current best practice is to prioritize the resource allocation. Therefore, each class is assigned a unique type of profit function, which has an implication of QoS differentiation. Assigning profit functions to classes to form a class-aware scheduling policy becomes a challenge to tackle. In this research, non-real-time packets are assumed receives lowest priority. Therefore, with two time-sensitive classes, only 12 scheduling policies in total are studied as shown in Table 4. 
Table 3 Definitions of Profit Functions

\begin{tabular}{|c|c|c|c|}
\hline Step-Down & & & \\
\hline Profit & Condition & & \\
\hline 1 & $T_{d}<=T_{b}$ & & \\
\hline 0 & $T_{d}>T_{b}$ & 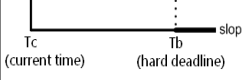 & $\stackrel{\text { time }}{\longrightarrow}$ \\
\hline Slope-Down & & & \\
\hline Profit & Condition & & \\
\hline 1 & $T_{d}<=T_{a}$ & protit & \\
\hline $1-\left(T_{d}-T_{a}\right)\left|S_{2}\right|$ & $T_{b}>T_{d}>=T_{a}$ & & \\
\hline 0 & $T_{d}>T_{b}$ & $\begin{array}{ccc}\mathrm{Tc} \\
\text { (current time) } \\
\text { (soft deadline) }\end{array}$ & $\underset{\substack{\longrightarrow \text { barime } \\
\text { (hard deadline) }}}{\longrightarrow}$ \\
\hline Slope-Step & & & \\
\hline Profit & Condition & profit & \\
\hline $1+\left(T_{b}-T_{d}\right)\left|S_{l}\right|$ & $T_{d}<=T_{b}$ & 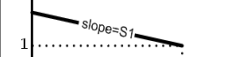 & \\
\hline 0 & $T_{d}>T_{b}$ & $\underset{\substack{\text { (curent time) } \\
\text { (hard deadline) }}}{\mathrm{Tc}}$ sio & $\longrightarrow$ time \\
\hline Double-Slope & & & \\
\hline Profit & Condition & profit & \\
\hline $1+\left(T_{a}-T_{d}\right)\left|S_{l}\right|$ & $T_{d}<=T_{a}$ & & \\
\hline $1-\left(T_{d}-T_{a}\right)\left|S_{2}\right|$ & $T_{b}>=T_{d}>T_{a}$ & & \\
\hline 0 & $T_{d}>T_{b}$ & $\begin{array}{cc}\mathrm{Tc} \\
\text { (current time) }\end{array} \quad \begin{array}{c}\mathrm{Ta} \\
\text { (soft deadline) }\end{array}$ & 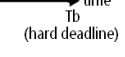 \\
\hline
\end{tabular}

Table 4 Scheduling Policies

\begin{tabular}{|c||l|l|}
\hline Policy & Conversational & Streaming \\
\hline $\mathbf{1}$ & Step-Down & Slope-Step \\
\hline $\mathbf{2}$ & Step-Down & Slope-Down \\
\hline $\mathbf{3}$ & Step-Down & Double-Slope \\
\hline $\mathbf{4}$ & Slope-Step & Slope-Down \\
\hline $\mathbf{5}$ & Slope-Step & Double-Slope \\
\hline $\mathbf{6}$ & Slope-Down & Double-Slope \\
\hline $\mathbf{7}$ & Slope-Step & Step-Down \\
\hline $\mathbf{8}$ & Slope-Down & Step-Down \\
\hline $\mathbf{9}$ & Double-Slope & Step-Down \\
\hline $\mathbf{1 0}$ & Slope-Down & Slope-Step \\
\hline $\mathbf{1 1}$ & Double-Slope & Slope-Step \\
\hline $\mathbf{1 2}$ & Double-Slope & Slope-Down \\
\hline
\end{tabular}

\subsection{Look-Ahead Scheduling}

The timeliness of a packet is affected by every router in its traveling path. Therefore, it is beneficial to examine the loading status of the succeeding routers in packet scheduling. For simplicity, this research uses latency-to-destination stored in the routing table to estimate the latency for the packet to reach its destination. With this estimated latency, the scheduler is able to assess the end-to-end timeliness of a packet and then adjust its scheduling decision accordingly as shown in Fig. 7. We can easily calculate the Expected Overdue Latency $(E O L)$ of a packet from its traveling schedule and its latency-to-destination. The hard deadline (if it is Step-Down or Slope-Step type) or the soft deadline (if it is Slope-Down or Double-Slope type) is moved backward by a fraction of EOL, called deadline adjustment coefficient. The same IRS algorithm is then applied to schedule the packet. Currently, there is no theoretical study to determine the deadline adjustment coefficient and has to be determined empirically.

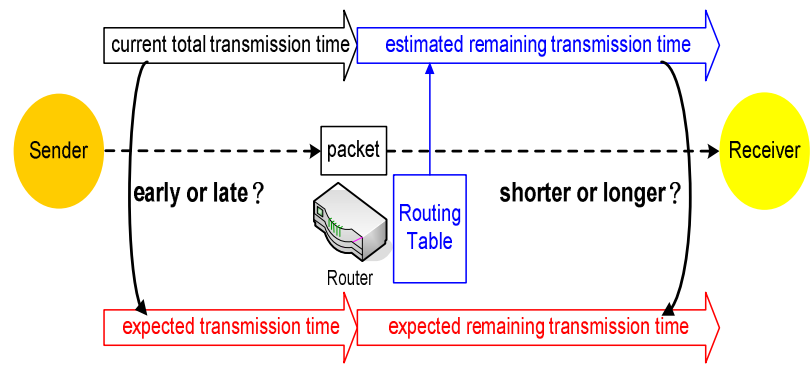

Fig. 7 Profit Function Adjustment for LAS

\section{PERFORMANCE EVALUATION}

The proposed scheduling method is evaluated using NS-2 network simulator [9].

\subsection{Environment of Experiments}

The linear topology used in Experiment 1 and 2 is shown in Fig. 8. The bandwidth and delay time of each link is $5 \mathrm{Mbps}$ and $20 \mathrm{~ms}$ respectively. There are several CBR streams simulating real-time traffic and a FTP stream simulating non-real-time traffic. Each router has a cross traffic of non-real-time traffic simulating traffic turbulence. 


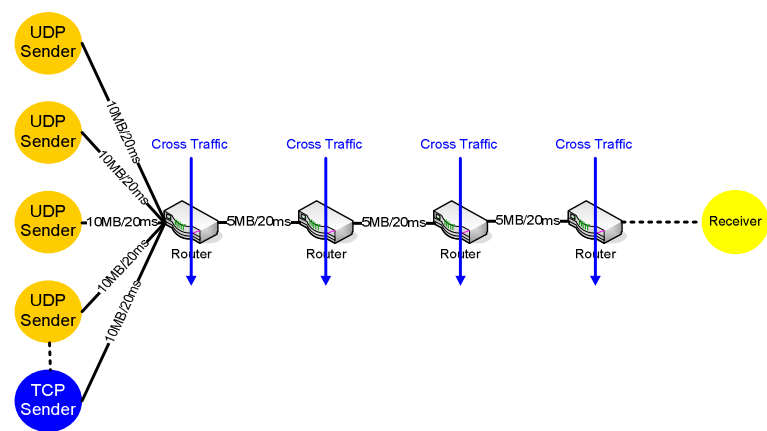

Fig. 8 Topology of Experiment 1 and 2

\subsection{Design of Experiments}

Experiment 1 and 2 studies the behavior of the proposed approach over different scheduling architectures when the traffic consists of only one real-time class and one non-real-time class. Experiment 1 studies the performance of IRS scheduling while Experiment 2 compares the IRS and LAS scheduling over PMQ architecture. The evaluation metrics for Experiment 1 and 2 are delay, jitter, and lost rate. Controlling variables are traffic load (number of CBR flows), network size (hop count) and queue size as shown in Table 5. Experiment 3 evaluates 12 class-aware scheduling policies as well as Priority Queue approach. The evaluation metric for Experiment 3 is total charge.

Table 5 Parameters used in Experiment 1 and 2

\begin{tabular}{|l|c|c|}
\hline \multicolumn{1}{|c|}{ Parameter } & $\begin{array}{c}\text { Values } \\
\text { if variant }\end{array}$ & $\begin{array}{c}\text { Values } \\
\text { if fixed }\end{array}$ \\
\hline $\begin{array}{l}\text { Number of CBR flows } \\
\text { @448 kbps per flow }\end{array}$ & $3,6,9,12,15,18$ & 12 \\
\hline Hop Count & $3,4,5,6,7,8$ & 4 \\
\hline Queue Size (packets) & $15,20,25,30,35,40$ & 20 \\
\hline
\end{tabular}

\subsection{Experiment 1}

FIFO queue is added to the experiment as the baseline performance. The experiment shows that SPQ is the best and MQ is the worst among all three architectures as we anticipated. All three architectures are better than FIFO queue. Fig. 9 shows part of experiment results when Step-Down profit function is used and the control variable is the number of CBR flows.

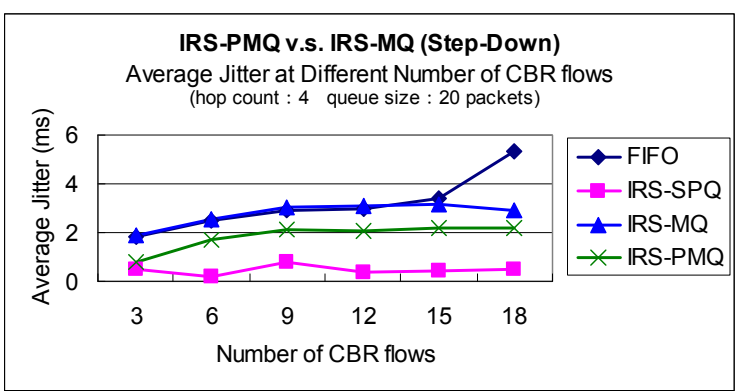

(a)

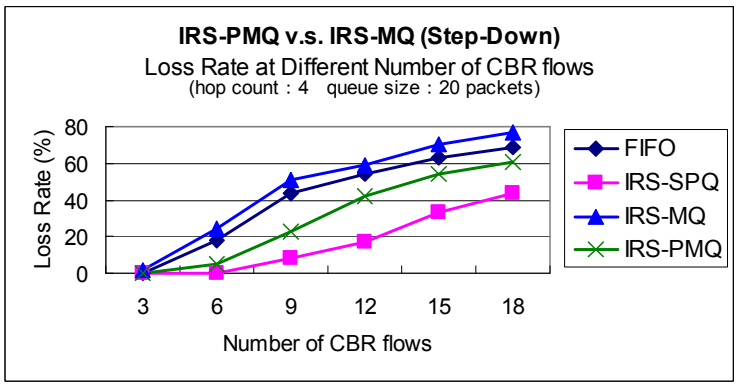

(b)

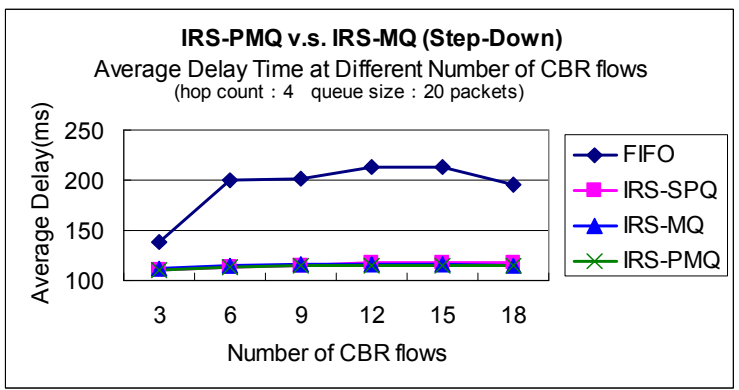

(c)

Fig. 9 Experiment 1: IRS

(a)jitter, (b)loss rate, (c)delay time.

\subsection{Experiment 2}

Experiment 2 compares the performance of LAS and IRS when single real-time class is used over PMQ scheduling architecture. The experiment shows that LAS outperforms IRS by a very large margin. Fig. 10 shows part of experiment results when Step-Down profit function is used and the control variable is the number of CBR flows. The deadline adjustment coefficient $(c)$ for LAS, is set at 0.1 and 0.2. 


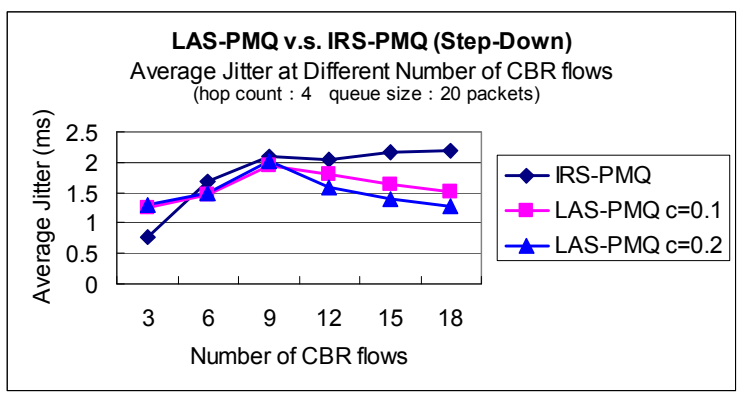

(a)

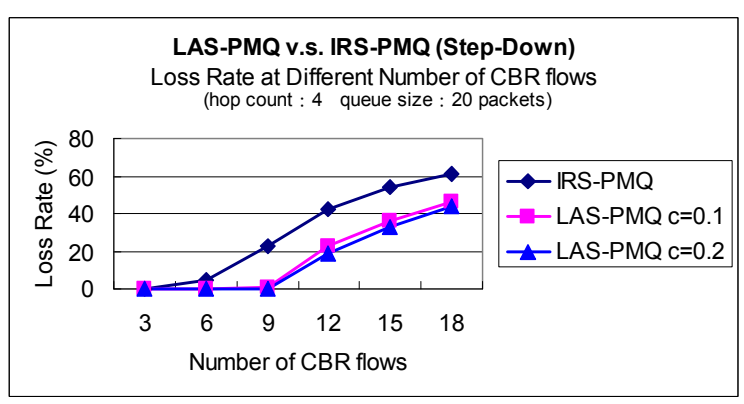

(b)

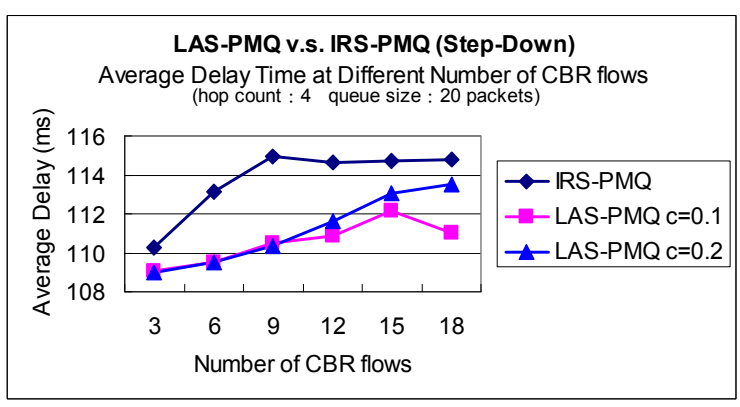

(c)

Fig. 10 Experiment 2: LAS vs. IRS

(a)jitter, (b)loss rate, (c)delay time.

\subsection{Experiment 3}

Experiment 3 evaluates 12 scheduling policies as well as simulated Priority Queue (SimPQ) approach [6]. To simulate Priority Queue, one queue is used for each class. The linear topology similar to the one used in Experiment 1 and 2 is shown in Fig. 11. The bandwidth and delay time of each link is $3 \mathrm{Mbps}$ and $20 \mathrm{~ms}$ respectively. The evaluation metric is the total charge as formulated in Eq. 1. Related parameters are shown in Table 6.

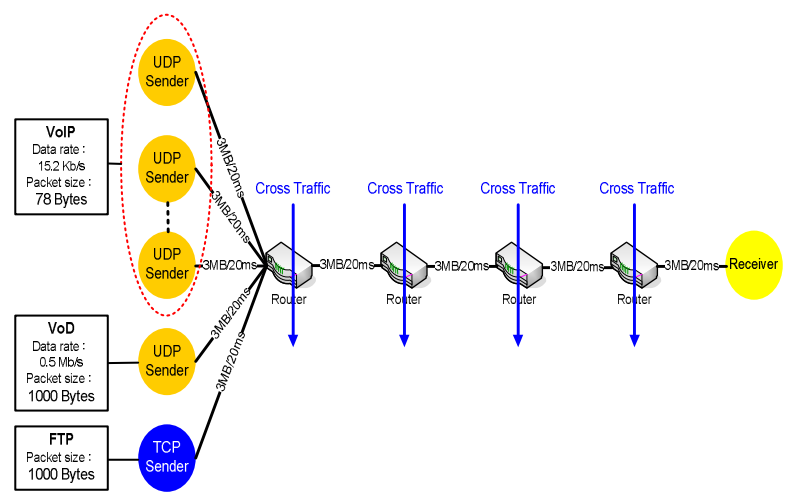

Fig. 11 Topology of Experiment 3

Table 6 Parameters used in Experiment 3

\begin{tabular}{|c|c|c|c|}
\hline & & Conversational & Streaming \\
\hline Class & $i$ & 1 & 2 \\
\hline \multirow{3}{*}{$\begin{array}{l}\text { Quality } \\
\text { coefficient }\end{array}$} & $a_{i}$ & 0.6 & 0.8 \\
\hline & $b_{i}$ & 0.2 & 0.0 \\
\hline & $c_{i}$ & 0.2 & 0.2 \\
\hline \multirow{3}{*}{$\begin{array}{l}\text { Charge } \\
\text { Threshold }\end{array}$} & $B_{i}(1)$ & $150 \mathrm{~ms}$ & $1 \mathrm{~min}$ \\
\hline & $B_{i}(2)$ & $15 \mathrm{~ms}$ & N.A. \\
\hline & $B_{i}(3)$ & 0.05 & N.A. \\
\hline \multirow{2}{*}{ Tariff } & $C_{1}, C_{2}$ & 0.001 & 0.0003 \\
\hline & \multicolumn{3}{|c|}{$C_{3}$ (Non-real-time Class) $=0.0001$} \\
\hline $\begin{array}{l}\text { No. of } \\
\text { flows }\end{array}$ & $N_{i}$ & $15,20,25,30,35,40$ & 1 \\
\hline
\end{tabular}

The results of Experiment 3 are shown in Fig. 12. As we can see, when the number of VoIP streams increases, SimPQ is not able to adjust its scheduling policy based on packet's timeliness while many of our policies can. From Fig. 12 we can see that Conversational class had better be assigned with either Slope-Down or Double Slope profit function. Policy 6 performs the best among all policies. 


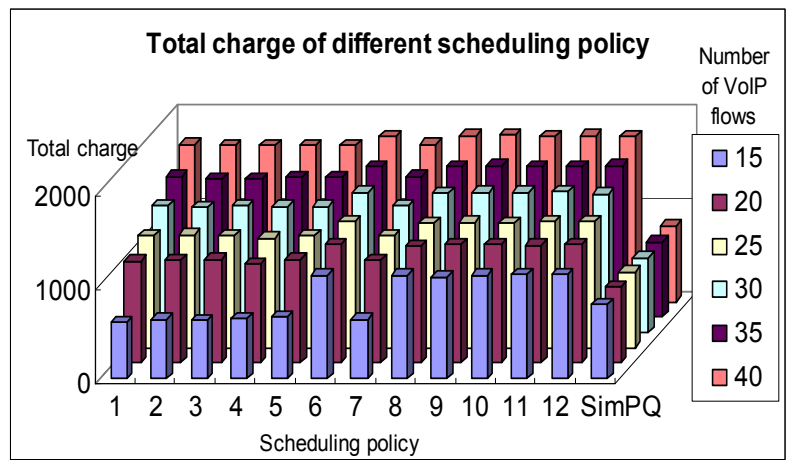

Fig. 12 Experiment 3: Multi-Class Scheduling Policy

\section{CONCLUDING REMARKS}

This paper presents a new packet scheduling approach that takes into account both the timeliness and QoS class of a packet to insert an outgoing packet into the "best" queuing position aiming to maximize the overall satisfaction factor, which is represented by a tariff formula that is sensitive to both QoS class and traffic volume. Routers are assumed in three different queue architectures: single preemptive queue and multiple FIFO queue with and without a priority queue. To forward a packet, a router first assigns an adequate profit function to the packet based on its timeliness and QoS class as well as the loading status in its succeeding routers along its predefined traveling path, and then inserts the packet into an appropriate queueing position. Our approach is evaluated by simulation using NS-2 network simulator. Simulation results show that our approach can outperform simulated Priority Queue by at least $34 \%$ in our evaluation metrics under heavy load. This research demonstrates that taking both timeliness and QoS class into account in packet scheduling is beneficial.

\section{REFERENCE}

1. Mohamed Ashour and Tho Le-Ngoc, "Performance Analysis of Weighted Fair Queues with Variable Service Rates," ICDT 2006.

2. C. Dovrolis, D. Stiliadis and P. Ramanathan, "Proportional Differentiated Services: Delay Differentiation and Packet scheduling," SIGCOMM 2002.

3. S. Floyd and V. Jacobson, "Link-sharing and resource management models for packet networks," IEEE/ACM Transactions on Networking, 3(4), (1995) 365-386.

4. Yao-Nan Lien, Hung-Ching Jang, Tsu-Chieh Tsai, and Hsing Luh, "Budget Based QoS
Management Infrastructure for All-IP Networks," Proc. of ICACT, vol. 1, Feb. 2005, pp. 185-190.

5. P. Lorenz, "Quality of service and new architectures for future telecommunications networks," Proc. of MILCOM, vol. 2, Oct. 2000, pp. 695-698.

6. S.-W. Moon, K.G. Shin, and J. Rexford, "Scalable Hardware Priority Queue Architectures for High-Speed Packet Switches," Proc. of RTAS, Jun. 1997, pp. 203-212.

7. D. Stiliadis, and A. Varma, "Latency-rate servers: a general model for analysis of traffic scheduling algorithms," IEEE/ACM Transactions on Networking, 6 (5), 1998, 611-624.

8. $\mathrm{Yu}$ Zhang, and Peter G. Harrison, "Performance of a Priority-Weighted Round Robin Mechanism for Differentiated Service Networks," Int'l Workshop on Performance Modeling and Evaluation in Computer and Telecommunication Networks (PMECT07).

9. "The Network Simulator - ns-2," http://www.isi.edu/nsnam/ns/. 